\title{
METODOLOGIA DE PROJETO DE UM CENTRO DE RECICLAGEM VIDRO ZEB EM BRASÍLIA,DF.
}

\author{
FARIA, Roberta C. A. \\ Universidade de Brasília, e-mail: robertacfaria7@gmail.com \\ SILVA, Caio F. E. \\ Universidade de Brasilia, e-mail: caiosilva@unb.br
}

\begin{abstract}
RESUMO
Devido à necessidade de se repensar a dinâmica de descarte de vidro no Distrito Federal, foi desenvolvido um projeto de arquitetura de um Centro de Reciclagem de Vidro. O projeto visa minimizar a dependência de energia do edifício por meio de um design arquitetônico adaptado ao clima local, tornando-se um edifício de balanço energético nulo - Zero Energy Building-ZEB. Neste sentido, o objetivo deste artigo é apresentar a metodologia do projeto de um edifício industrial ZEB. Para o desenvolvimento do projeto utilizou-se uma metodologia de simulação computacional com o software DesignBuilder (v5.5) e Revit (2019) desde as etapas iniciais de projeto. Esses programas permitem avaliações de cunho termoenergético, lumínico e volumétrico possibilitando testar alternativas que melhorem o desempenho do edifício. Os resultados obtidos com o projeto mostram que o galpão industrial projetado possui $81 \%$ de horas ocupadas em conforto, cerca de $80 \%$ da área é iluminada naturalmente durante o dia e houve redução de $71 \%$ da demanda energética. Destaca-se que no caso do Centro de Reciclagem de Vidro o uso de módulos fotovoltaicos devido à alta incidência de radiação solar em Braślia, logo, a produção de energia local abastece $94 \%$ da demanda energética do edifício. Sendo assim, com a integração de tecnologias renováveis ao projeto de arquitetura com baixa demanda energética foi possível obter o projeto de edifício industrial com características ZEB para o contexto climático do Distrito Federal.
\end{abstract}

Palavras-chave: Sustentabilidade, Simulação Computacional, Eficiência energética, Inovação e ZEB.

\begin{abstract}
Due to the necessity of rethink the glass waste dynamic in Distrito Federal, was developed an architectural project of a Center of Glass Recycling with low energy demand (ZEB). The main goal of this project is minimized building energy consumption through passive designs adapted for local climate. For the project development it was used computer simulation as a methodology with DesignBuilder (v5.5) and Revit (2019) since early design. Those softwares allowed testing several design alternatives by thermal energetic, natural lighting and volume validations that could improve the building performance. The industrial hangar's project outcomes are $81 \%$ hours occupied with comfort, $80 \%$ of area with natural lighting during the day and $71 \%$ reduction of energy consumption. It is highlighted the installation of photovoltaic modules at the Integrated Center of Glass Recycling, due to the high solar radiation incident in Brasilia during in major part of the year. Therefore, the local energy production supplies $94 \%$ of the energetic building demand. In summary, an architectural project with low energy demand integrated with renewable technology could provide ZEB constructions in Distrito Federal.
\end{abstract}

Keywords: Sustainability, Computer Simulation, Energy Efficiency, Innovation and ZEB.

FARIA, R. C. A.; SILVA, C. F. E. Metodologia projetual de um centro de reciclagem de vidro ZEB em Brasilia, DF. In: SIMPÓSIO BRASILEIRO DE QUALIDADE DO PROJETO NO AMBIENTE CONSTRUÍDO, 6., 2019, Uberlândia. Anais... Uberlândia: PPGAU/FAUeD/UFU, 2019. P 692-702. DOI https://doi.org/10.14393/sbqp19064. 


\section{INTRODUÇÃO}

O espaço construído surge como proteção dos indivíduos e animais no meio natural. Neste sentido, a arquitetura sustentável representa uma evolução deste conceito em que fomenta a construção de comunidade mais sustentável, ligados ao objetivo 11 dos Objetivos de Desenvolvimento Sustentável - ODS (ONU, 2019). Segundo Romero (2015), ao considerar somente as variáveis bioclimáticas, o meio ambiente manifesta-se de forma hostil para o homem e isso decorre da presença de fatores como temperatura, umidade, radiação solar, ventos e outros. Sendo assim, o uso da arquitetura como forma de proteção do homem ao meio, consequentemente, a torna sustentável pois a construção se adapta as variáveis específicas de onde está sendo alocada. Aspectos como dados climáticos, evolução biológica e soluções tecnológicas são levados em conta ao se trabalhar com a adaptação da arquitetura ao clima. Dados climáticos são as características anuais as variáveis presentes do meio (temperatura, umidade, ventos, etc), já a evolução biológica é o aspecto que diagnóstica o nível ideal de conforto do usuário, e as soluções tecnológicas são os métodos calculáveis para posteriormente se aplicar na arquitetura. (OLGYAY; OLGYAY, 1963,). A atual demanda de se produzir arquitetura está voltada para a conservação do meio ambiente, principalmente na questão do consumo energético e emissões de gases de efeito estufa (GEE), tendo em vista que um edifício, durante sua vida útil, pode consumir cerca de até $50 \%$ da matéria prima encontrada na natureza. (RUUSKA; HAKKINEN, 2014). Corbellas e Yanna (2009) comentam que a adequada associação do edifício ao entorno promove uma redução do consumo energético do mesmo. Tendo em vista a construção de edifícios com baixa carga energética surge o conceito Zero Energy Building, que de acordo com Torcellini (2006), um edifício ZEB, seja qual for sua tipologia, têm uma significativa redução no consumo de energia e um balanço energético baixo quando aplicada tecnologias renováveis. Para dar suporte ao projeto de um edifício com boa performance energética é importante que se faça o uso de simulação computacional, pois pode-se gerar um modelo aproximado da realidade que prevê o comportamento do projeto em diversos aspectos. (PETERS; PETERS, 2018). Em relação aos métodos de avaliação, a introdução da simulação computacional desde as fases iniciais do projeto de arquitetura é algo complexo, contudo essencial para se obter decisões mais assertivas para uma boa performance do edifício (OSTERGARD; JENSEN; MAAGAARD, 2016). No Brasil, Lamberts et al. (2005) discutem que há pouco incentivo na obtenção de construções com baixo consumo energético, e que isso decorre da falta de iniciativas governamentais que fomentem a utilização de mais ferramentas de simulação durante o projeto.

Tendo em vista o quadro nacional com poucos exemplos de edifícios com características ZEB, o objetivo desse trabalho é propor uma metodologia de projeto de um Centro de Reciclagem de Vidro ZEB no Distrito Federal, utilizando tecnologia de simulação computacional durante todo o processo projetual.

A escolha da capital federal como base climática para o estudo decorre do grande potencial para instalação de edificações de balanço energético nulo devido a sua temperatura, humidade relativa do ar e irradiância solar global (DOMINGOS et al., 2014). Dentro do processo projetual desse Centro foram feitas simulações computacionais, desde o estudo preliminar, com os softwares 
Revit 2019 para modelagem volumétrica e DesignBuilder v5.5 para avaliações de desempenho termoenergético e lumínico. O projeto abarca as atividades de reciclagem e produção do vidro, e a tipologia do projeto é de um galpão industrial, tendo em vista que o setor industrial é um grande consumidor de energia mundialmente.

\section{METODOLOGIA}

Para o desenvolvimento do projeto do Centro de Reciclagem de Vidro ZEB foram utilizados alguns procedimentos de simulação computacional, sendo eles a modelagem volumétrica computacional e física (impressora 3D), de conforto, de iluminação natural e termoenergética. O projeto é composto de três diferentes ambientes com a mesma tipologia modular de galpão industrial. Os ambientes estão separados em área de reciclagem e produção, área da comunidade inovação e de área dos funcionários (Figura 1). O Centro possui ao todo $15.428,15 \mathrm{~m}^{2}$ e o galpão de reciclagem e produção $9.438,75 \mathrm{~m}^{2}$.

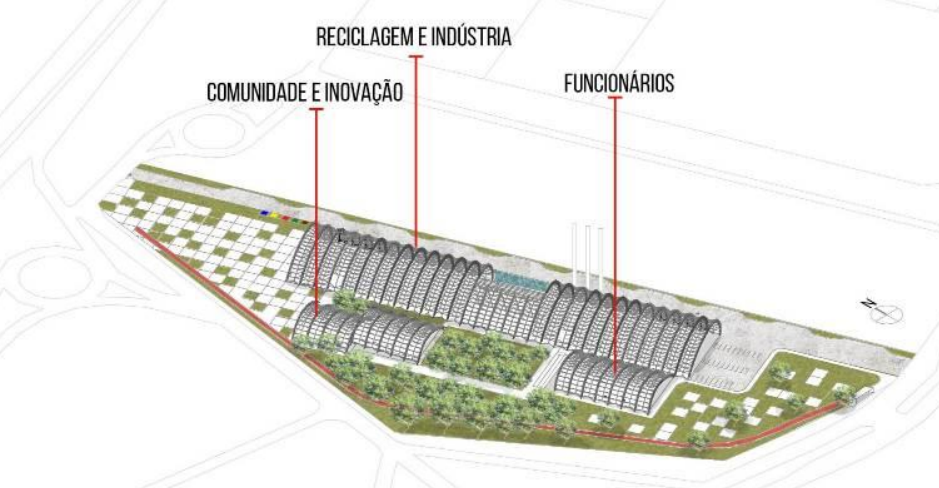

Figura 1 - Mapa de uso do Centro de Reciclagem de Vidro ZEB Fonte: Acervo pessoal dos autores (2018)

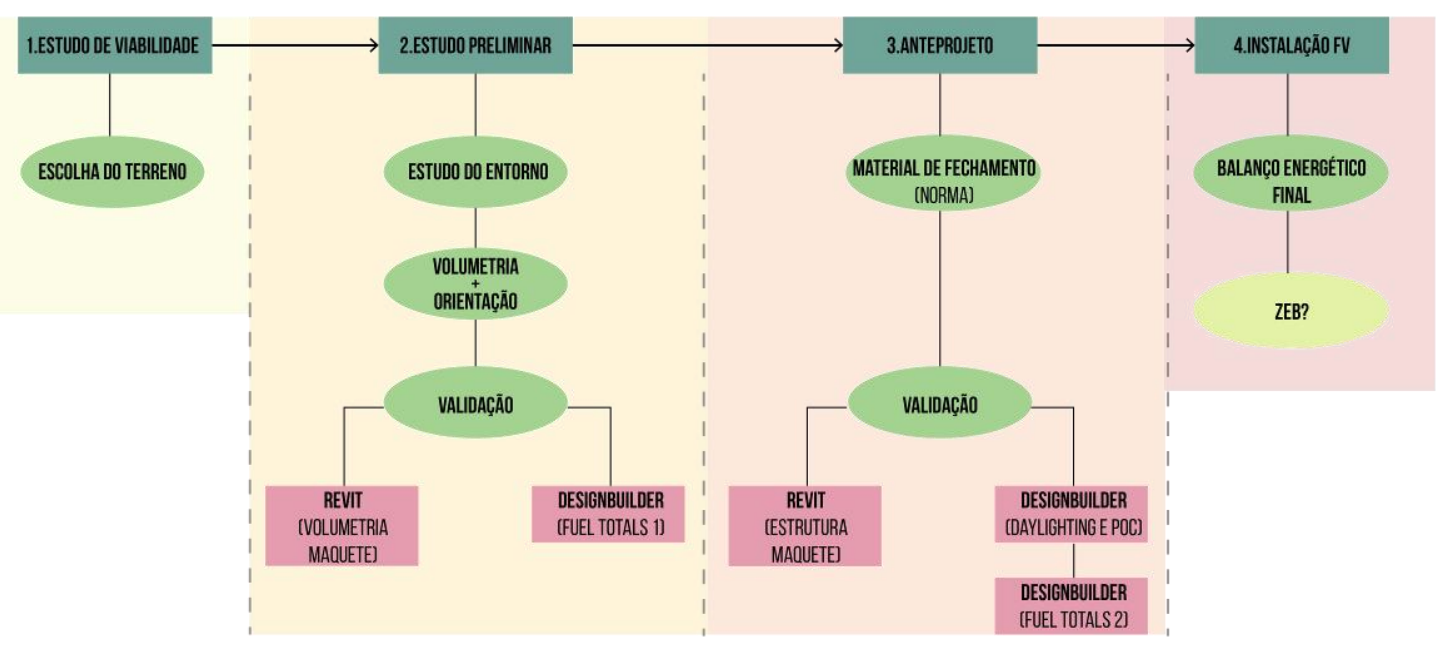

Figura 2 - Fluxograma do método de trabalho Fonte: Acervo pessoal dos autores (2019)

Para as análises volumétricas e espaciais foram considerados os três setores, no entanto, para os estudos termoenergético e lumínico foi dado enfoque no galpão de reciclagem e indústria para tentar obter resultados mais 
detalhados. A metodologia do projeto se divide em quatro etapas, sendo elas o estudo de viabilidade, o estudo preliminar, o anteprojeto e a instalação de fotovoltaica (FV), como apresentado na Figura 2.

O edifício está localizado no Setor de Indústrias e Abastecimento, na cidade de Brasília, Distrito Federal, o estudo de viabilidade do terreno hipotético avalia a norma de gabarito, o tráfego e os raios de influência. Na fase de estudo preliminar são analisados o entorno, a orientação do edifício e sua volumetria, para os dois últimos aspectos foi feita a validação com simulação volumétrica no Revit e simulação do consumo de energia inicial no DesignBuilder. A segunda etapa é de anteprojeto que avaliou a qualidade dos materiais de fechamento e da estrutura de apoio do módulo do galpão, sendo feita a validação da estrutura do galpão no Revit, da qualidade lumínica e de conforto no DesignBuilder e após a verificação dos materiais pode-se avaliar o consumo energético final do projeto, também, no DesignBuilder. Por fim, estipula-se a quantidade e qualidade dos módulos fotovoltaicos a serem instalados no projeto para que se faça o balanço energético final do edifício para saber se o mesmo atingiu ou não níveis ZEB.

\section{RESULTADOS}

Os resultados obtidos durante as análises feitas direcionam o projeto a alcançar um consumo baixo de energia, principalmente dada a tipologia aplicada de um Centro de Reciclagem de Vidro que propõe produzir embalagens de vidro a partir do vidro reciclado. A atividade existente nesse centro faz o uso de maquinários com elevado consumo energético, o que torna essencial que o edifício tenha um baixo consumo de energia para poder viabilizar a existência desse tipo de empreendimento sem gerar grandes impactos ambientais. E para isso foram feitos um estudo de viabilidade, o estudo preliminar, o anteprojeto e a instalação de fotovoltaicas.

\subsection{Estudo de Viabilidade}

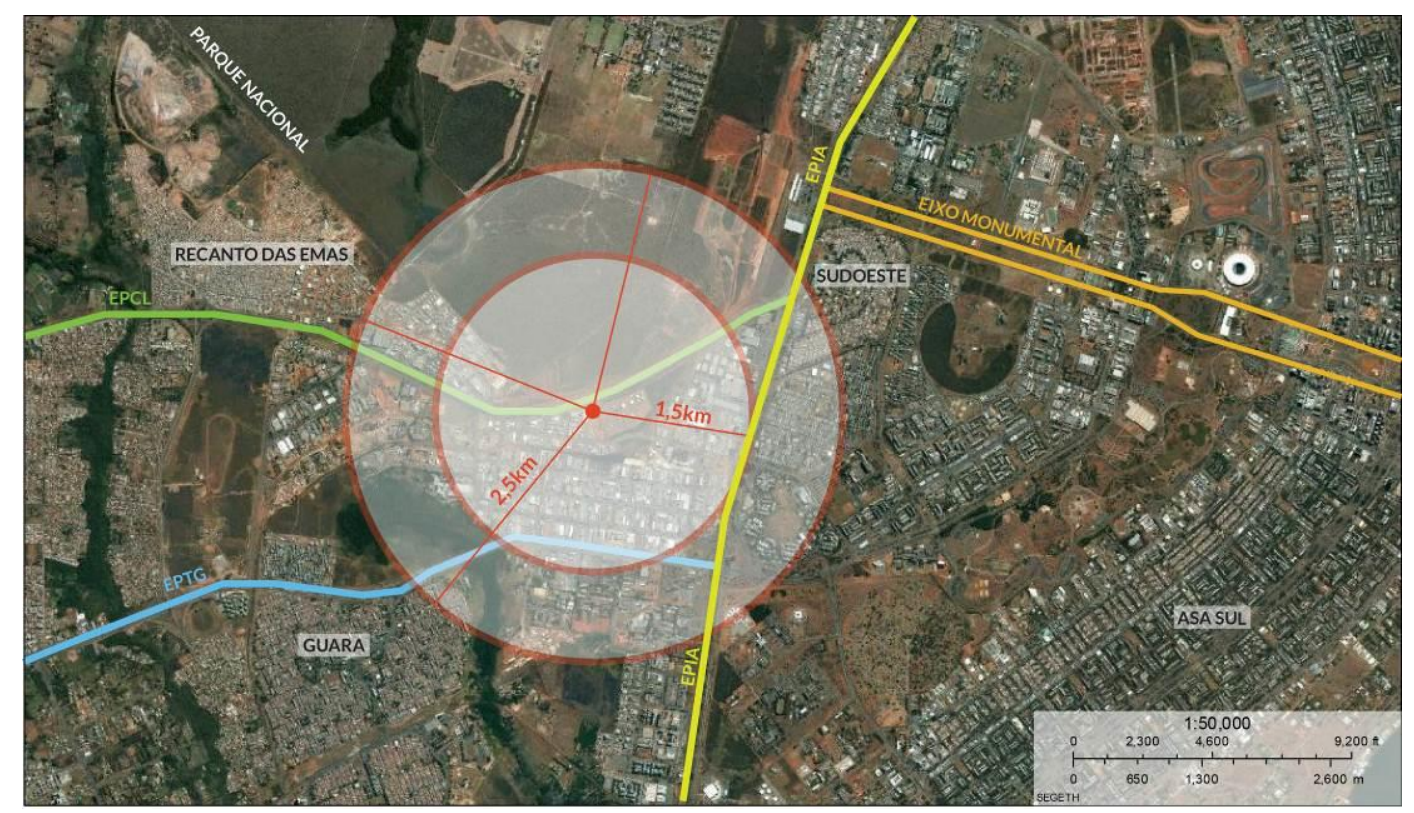

Figura 3 - Estudo da qualidade urbana -

Fonte: Geoportal (2018) 
No Estudo de viabilidade foi utilizado alguns critérios de escolha do terreno, sendo eles a aproximação com a via EPIA, que contorna todo o DF e se o lote permite o uso para fins econômicos. Após essa verificação utilizou-se a metodologia do Selo Casa Azul, elaborado pela Caixa Econômica Federal, para a avaliação da qualidade urbana. Esse estudo avalia os impactos do empreendimento em seu entorno imediato. Para isso aplica-se raios de 2,5km e 1,5km (Figura 3), a partir do terreno escolhido, para analisar os possíveis impactos causados pelo projeto na vizinhança.

Após o estudo do entorno e avaliação das normas de gabarito foi escolhido o terreno hipotético (Figura 4) para o projeto, que está perto de quatro importantes vias, sendo elas EPIA, Eixo Monumental, EPTG e EPCL, e possui $45.793,15 \mathrm{~m}^{2}$ de área total.

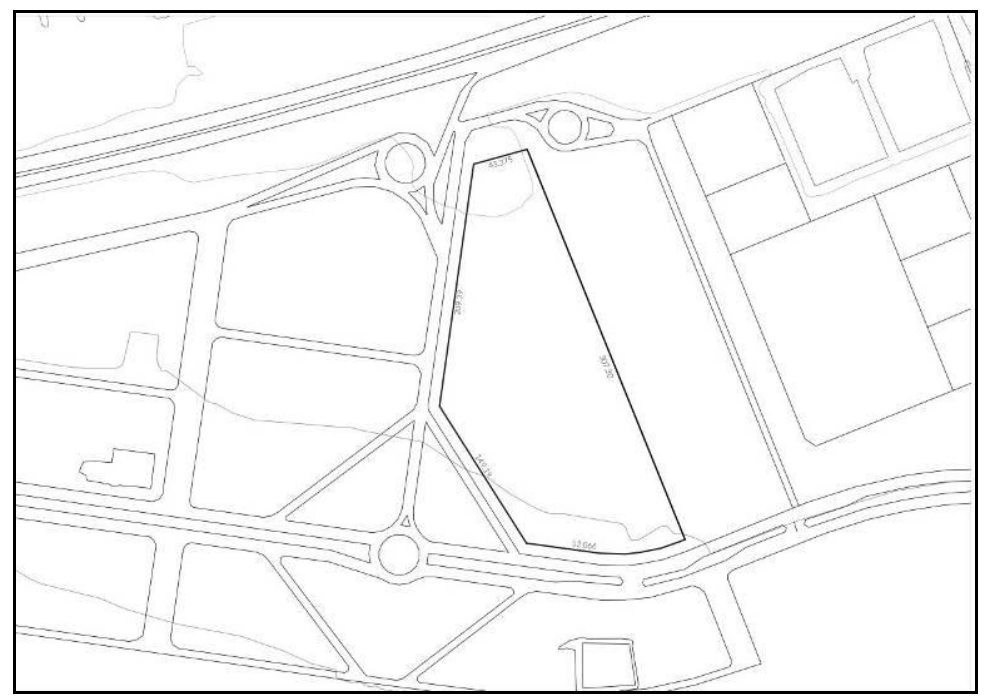

Figura 3 - Terreno Hipotético, SAI (Distrito Federal) -

Fonte: Acervo pessoal dos autores (2018)

\subsection{Estudo Preliminar}

No Estudo Preliminar foram avaliados o entorno imediato em suas características físicas (topografia, barreiras lindeiras e polvição sonora) e bioclimáticas para setorizar o projeto de maneira a valorizar os ventos predominantes e o percurso solar. Após esse estudo, são testadas três diferentes volumetrias e orientações para a implantação de um projeto de baixo consumo energético (Figura 5). Para isso, foi utilizado o software DesignBuilder que fez o estudo de fuel totals o qual avalia o parâmetro de consumo total de energia do modelo. O critério de escolha da volumetria base para um Centro ZEB de reciclagem de vidro foi a de menor consumo energético, sendo ela a opção de 3,5GWh correspondente à 233,34KWh/m².
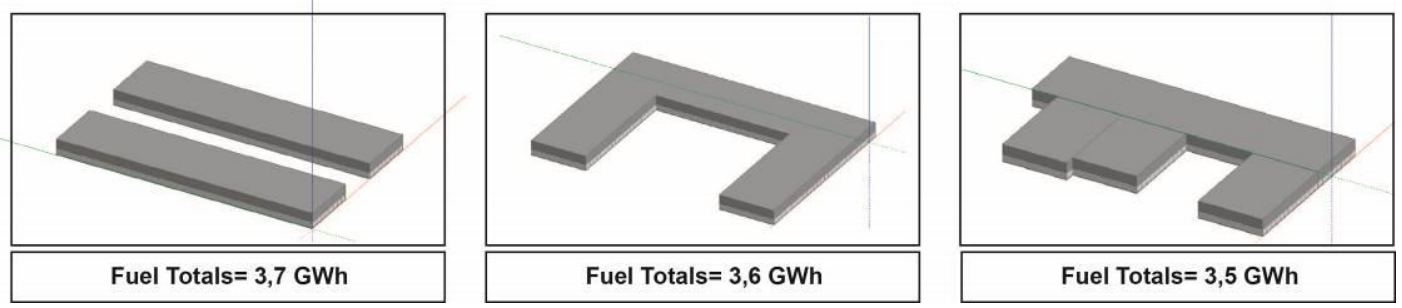

Figura 5 - Estudo Fuel Totals de volumetrias e orientações (validação) -

Fonte: DesignBuilder (2018) 
Também foi utilizado o software Revit para validar a espacialidade do Centro testando a proporção, a forma e a estética com tecnologia Building Information Modeling (BIM), que permite visualizar e atualizar o projeto a partir de plantas, cortes, vistas e volumetrias concomitantemente (Figura 6).

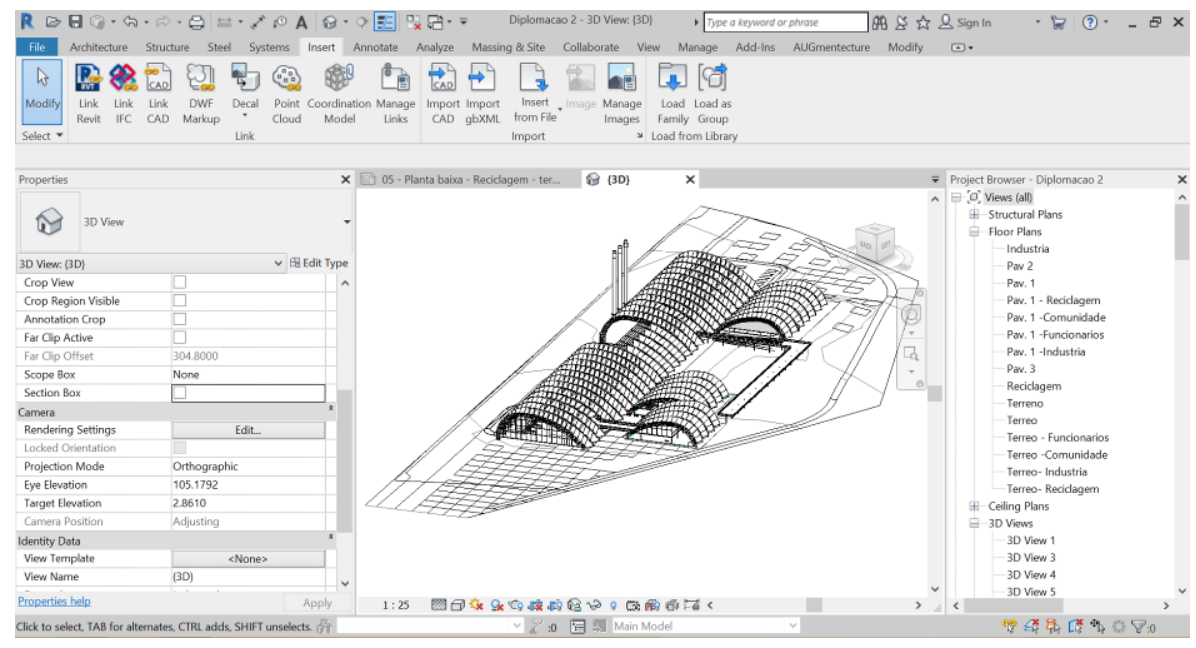

Figura 6 - Modelagem 3D no software Revit (validação) -

Fonte: Acervo pessoal dos autores (2019)

\subsection{Anteprojeto}

Para a fase de Anteprojeto utilizou-se a volumetria base escolhida no Estudo Preliminar e a partir dela o projeto foi se desenvolvendo com a especificação dos materiais de fechamento opaco e translúcido, consumo final de energia e a concepção estrutural do galpão. Inicialmente, os materiais de fechamento opaco (Figura 7) foram escolhidos a partir da norma NBR 15.220 para zona bioclimática 4, que é a de Brasília, possuindo transmitância de 1,65 W/m² K em sua composição e absortância de 0,3 com pintura branca (DORNELLES, 2008).

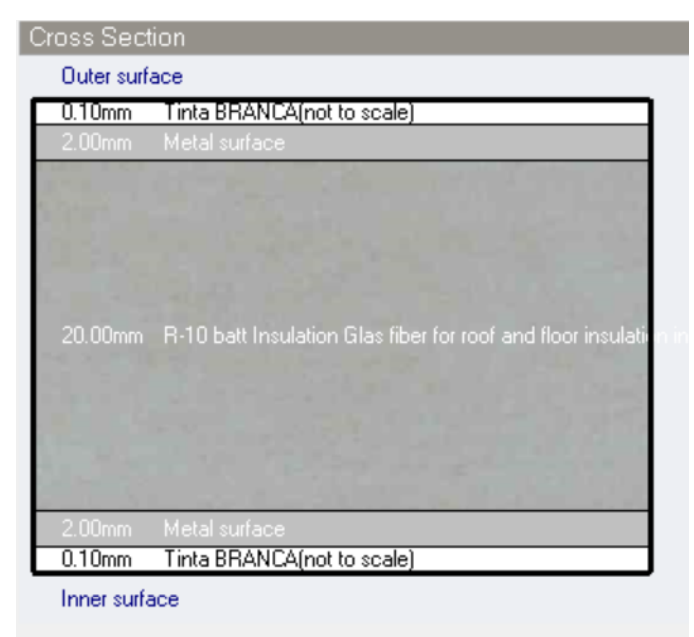

Figura 7 - Composição do material opaco de fechamento Fonte: DesignBuilder (2018)

\subsubsection{Daylighting (validação)}

Para o material de fechamento translúcido é feito o estudo de daylighting para verificar se a quantidade de aberturas zenitais propostas atende ao sugerido pela NBR 5413. Nesse estudo se utiliza o algoritmo de cálculo do 
Radiance dentro da interface visual do DesignBuilder, que extrai valores relativos ao daylight factor gerando um mapa gráfico com a quantidade de lux refletido na superfície do piso (Figura 8).
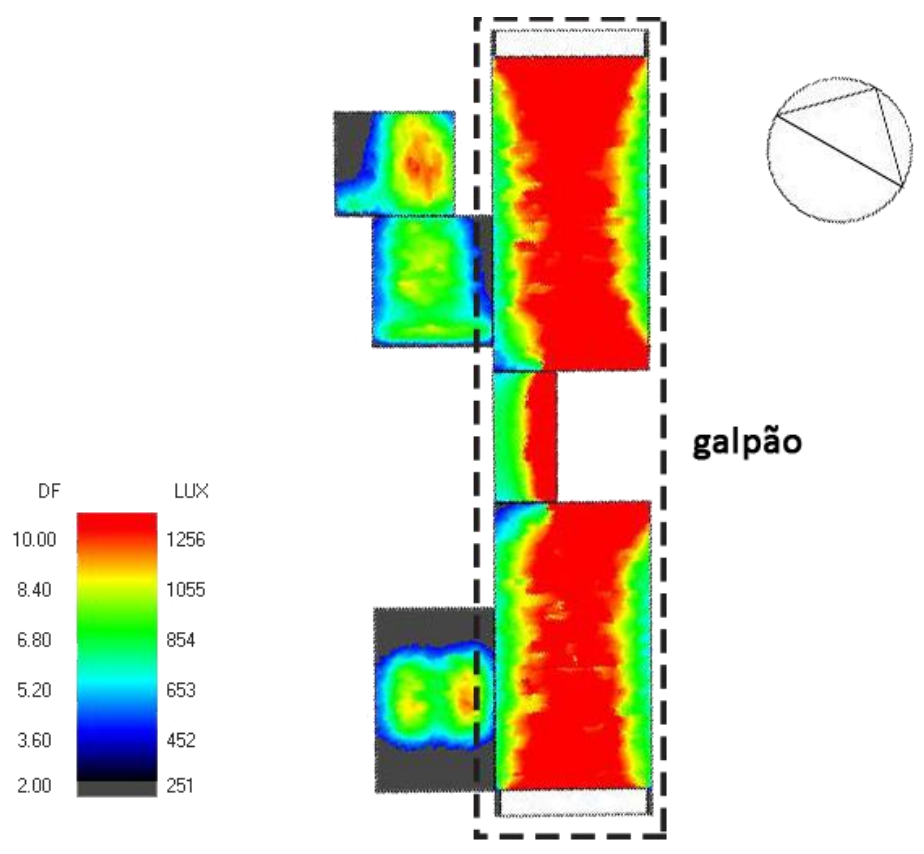

Figura 8 - Análise de Daylighting -

Fonte: DesignBuilder (2018)

O resultado obtido da análise de daylighting mostra que mais de $80 \%$ da área do galpão recebe cerca de 1256 lux, o que está de acordo com a NBR 5413, que sugere para tipologias de galpão industrial (classe B) o valor entre 10002000lux.

\subsubsection{Percentual de Horas Ocupadas em Conforto (validação)}

Após a avaliação de daylighting, foi testado dois tipos de vidro, sendo eles um simples de $3 \mathrm{~mm}$ e um duplo de $6 \mathrm{~mm}$. O teste valida o Percentual de Horas Ocupadas em Conforto (POC), pois para o projeto pretende-se utilizar $\mathrm{O}$ máximo potencial de iluminação natural sem prejudicar o conforto térmico interno. Para o estudo de POC, o software DesignBuilder avaliou 8.760 horas no ano, com o uso do modelo adaptativo de De Dear e Brager (2002) a partir da fórmula de temperatura neutra abaixo e utilizando um voto médio predito (PMV) de $+2,5^{\circ} \mathrm{C}$.

$$
\mathrm{TN}=17,8+0,310 \mathrm{Tar}
$$

onde, $\mathrm{TN}=$ temperatura neutra $\left({ }^{\circ} \mathrm{C}\right)$;

Tar $=$ temperatura do $\operatorname{ar}\left({ }^{\circ} \mathrm{C}\right)$.

De acordo com Assis e Pereira (2010), alguns autores estão adotando índices adaptativos de forma mais simples, em fases iniciais de projetos, devido a dificuldade de estimar um PMV em climas tropicais.

O resultado obtido foi que com o uso do vidro duplo de $6 \mathrm{~mm}$ no projeto atinge $81 \%$ de horas ocupadas em conforto (Quadro 1), o que de acordo com o Regulamento Técnico da Qualidade para o Nível de Eficiência Energética de Edificações Comerciais, de Serviços e Públicas (RTQ-C), equivale a uma etiquetagem A para o quesito avaliado das envoltórias. 
Quadro 1 - Comparativo do Percentual de Horas Ocupadas em Conforto

\begin{tabular}{|c|c|}
\hline Vidro simples 3mm & Vidro duplo 6mm \\
\hline $\mathrm{U}=5,70 \mathrm{~W} / \mathrm{m} 2-\mathrm{k}$ & $\mathrm{U}=3,03 \mathrm{~W} / \mathrm{m} 2-\mathrm{k}$ \\
\hline 6955 horas de conforto & $\mathbf{7 0 9 4}$ horas de conforto \\
\hline $\mathbf{7 9 \%}$ & $\mathbf{8 1 \%}$ \\
\hline \multicolumn{2}{|c|}{ Fonte: DesignBuilder (2018) e Fórmula de De Dear e Brager (2002) } \\
\hline
\end{tabular}

\subsubsection{Análise Fuel Totals final (validação)}

Após analisar as características de daylighting e conforto foi feita a simulação referente ao consumo energético do projeto denominada Fuel Totals. Nessa simulação foram estabelecidos alguns parâmetros para o projeto, sendo eles apresentado no quadro 2 abaixo:

Quadro 2 - Parâmetros de análise Fuel Totals

\begin{tabular}{|c|}
\hline Parâmetros de simulação \\
\hline Iluminação artificial desligada no galpão \\
\hline Ventilação ligada \\
\hline Ar condicionado desligado \\
\hline Transmitância chapa metálica: U= 1,65 W/m2-K (NBR 15.220) \\
\hline Vidro duplo $6 \mathrm{~mm} / 6 \mathrm{~mm} / \mathrm{ar}$ \\
\hline Fonte: Acervo pessoal dos autores
\end{tabular}

Esses parâmetros só puderam ser e determinados por causa das simulações atribuídas anteriormente, que validaram as especificações feitas no projeto. Após a análise de Fuel totals com os parâmetros estabelecidos acima (Quadro 2) o resultado obtido foi de que o edifício consome 1.004.398,00KWh correspondente à $65,10 \mathrm{KWh} / \mathrm{m}^{2}$. O que significa que houve uma redução de $71 \%$ do consumo de energia quando comparada com o consumo obtido na volumetria base avaliada no Estudo Preliminar.

\subsubsection{Concepção estrutural (validação)}

A parte estrutural do projeto foi consolidada com a contribuição da utilização do software Revit associado com a tecnologia de impressora 3D. Com essas ferramentas pôde-se criar modelos interativos e físicos de maneira rápida para a validação da estrutura proposta, como apresentado nas figuras 9 e 10.

Essa validação ocorre com a impressão 3D das peças do módulo, como treliças, longarinas e chapas do fechamento, e a montagem da maquete em escala 1:250 avaliando as dificuldades de execução e sustentação das peças. 


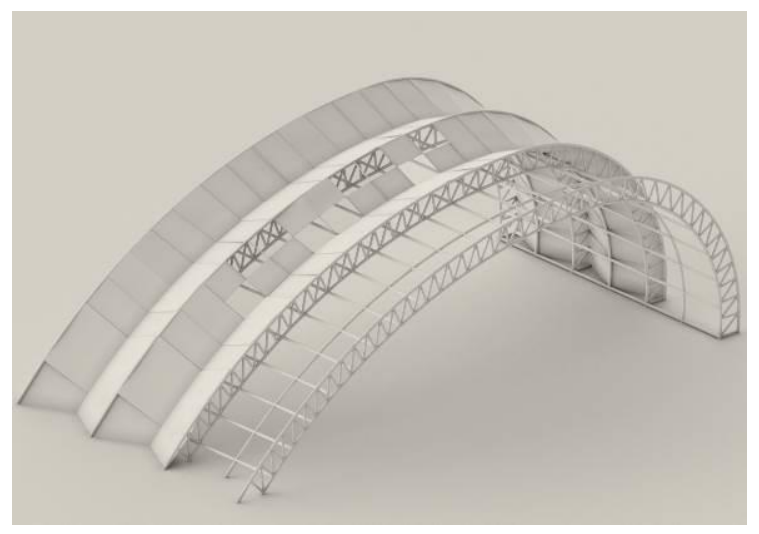

Figura 9 - Render do módulo estrutural Fonte: Revit (2018)

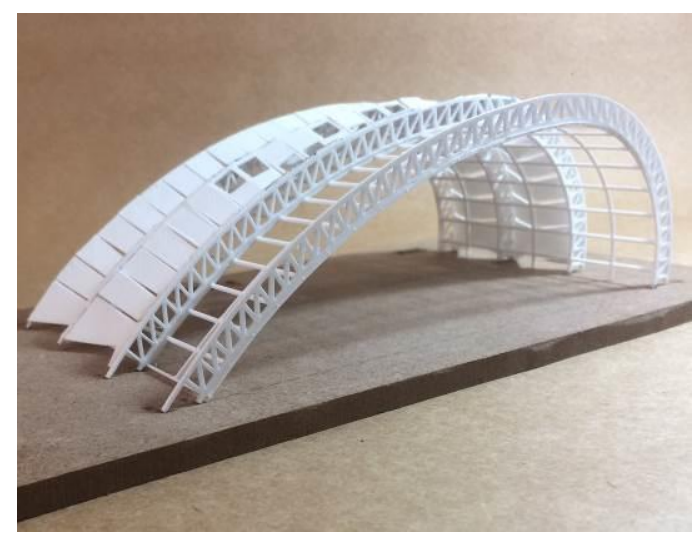

Figura 10 - Maquete física esc.1:250- impressa 3D Fonte: Acervo pessoal dos autores

\subsection{Instalação FV}

Quadro 3 - Estimativa de instalação de FV

\begin{tabular}{|c|c|}
\hline \multicolumn{2}{|c|}{ Fotovoltaicas } \\
\hline \multicolumn{2}{|c|}{1 Módulo - 405W produz 1,458 KWh } \\
\hline Qtd. Módulos & Produção KWh (Anual) \\
\hline 150 & $1.117 .557,00$ \\
\hline 500 & $3.725 .190,00$ \\
\hline 800 & $5.960 .304,00$ \\
\hline 1000 & $7.450 .378,00$ \\
\hline
\end{tabular}

Para tentar se aproximar do consumo energético real de uma indústria vidreira, foram considerados ao final do projeto o uso de maquinários específicos, como forno e paletizadoras, obtendo-se o consumo total final de 7.887.492,77KWh. Com a finalidade de se obter um edifício ZEB, foram utilizados módulos fotovoltaicos, considerados a melhor estratégia ativa de captação de energia limpa para o contexto climático de Brasília. Dessa forma, propondo o uso de 1.000 módulos de 405W de potência instalada (Quadro 3) ocupando 
$2.100 \mathrm{~m}^{2}$ da cobertura do galpão de reciclagem o projeto atinge um balanço energético final de 94\%, o que, a partir dos dados apresentados pela CEB (2014), também pode corresponder ao abastecimento de 2.759 residências por ano no Distrito Federal.

\section{CONCLUSÕES}

Diminuir a demanda energética de uma construção já é uma realidade para produzir uma arquitetura adaptada as necessidades do meio natural. Dentre diferentes formas de se projetar, o uso da simulação integrada ao processo de projeto é uma estratégia adequada para se obter uma construção de mais qualidade e de menor impacto ambiental. Exemplo disso são os resultados apresentados no trabalho exposto, que ao utilizar a simulação computacional pode-se verificar o desempenho desse projeto antes mesmo de sua construção.

Do ponto de vista do uso de softwares, principalmente, daqueles voltados para avaliação termoenergética e lumínica desde o início do projeto, foi possivel determinar estratégias passivas no design que contribuíram para 0 desenvolvimento de uma arquitetura mais sustentável. Neste estudo, obtevese uma redução de $71 \%$ do consumo de energia inicial. Segundo Morbitzer et.al (2001), a simulação computacional pode focar em problemas setorizados de acordo com cada fase de projeto evitando que se estenda para as fases subsequentes. Os resultados também apontam a possibilidade de se gerar arquiteturas que funcionem como usinas de produção local de energia no contexto climático do Distrito Federal. Em resumo, a atribuição de ferramentas tecnológicas dentro do processo de projeto de arquitetura evidencia uma evolução na qualidade de construção das cidades com a possibilidade de diminuição do consumo de energia e emissão de gases polventes. Outro ponto em destaque é a utilização integrada de estratégias passivas e ativas com validação computacional no projeto para a geração de mais edifícios ZEB no contexto brasileiro.

\section{AGRADECIMENTOS}

Os autores agradecem ao grupo de pesquisa SiCAC (Simulação Computacional no Ambiente Construído - FAU/UnB) pelo apoio durante a pesquisa.

\section{REFERÊNCIAS}

ASSIS, E. S.; PEREIRA, I. M. Avaliações de modelos de índices adaptativos para uso no projeto arquitetônico bioclimático. Ambiente Construído, Porto Alegre, v. 10, n.1, p.31-51. 2010. Disponível em: <http://www.scielo.br/pdf/ac/v10n1/v10n1a02.pdf>.

ASSOCIAÇÃO BRASILEIRA DE NORMAS TÉCNICAS (ABNT). NBR-5413: Iluminância de Interiores - Referências - Elaboração. Rio de Janeiro, 1992.

ASSOCIAÇÃO BRASILEIRA DE NORMAS TÉCNICAS (ABNT). NBR-15220:

Desempenho térmico de edificações Parte 1: Definições, símbolos e unidades Referências - Elaboração. Rio de Janeiro, 2003. 
COMPAINHA ENERGÉTICA DE BRASÍLIA. CEB Distribuição S.A. In: XVI Encontro Nacional dos Conselhos de Consumidores de Energia Elétrica. 2014. Brasília, DF. 19 slides.

CORBELLA, O.; YANNAS, S. Em busca de uma Arquitetura Sustentável para os trópicos. Rio de Janeiro: Revan, 2009.

DOMINGOS, L.; KALZ, D.; DINKEL, A.; LOMARDO, L.; SILVA, V. Definição de uma classificação climática para o estudo de edificações com balanço anual zero de energia no Brasil. XV Encontro Nacional de Tecnologia do Ambiente construído, p.213-222, novembro de 2014, Maceió.

DORNELLES, K. A. Absortância Solar de superfícies opacas: Métodos de determinação e base de dados para tintas látex acrílica e PVA. Dissertação (Doutorado em Engenharia Civil) Faculdade de Engenharia Civil, Arquitetura e Urbanismo, Universidade Estadual de Campinas, Campinas, 2008.

INSTITUTO NACIONAL DE METROLOGIA, NORMALIZAÇÃO E QUALIDADE INDUSTRIAL (INMETRO). Regulamento Técnico da Qualidade do Nível de Eficiência Energética de Edifícios Comerciais, de Serviços e Públicos. Anexo da Portaria INMETRO nº 163/2009. 2009.

MORBITZER, C.; STRACHAN, P.; WESTER, J.; SPIRES, B.; CAFFERTY, D. Integration of Building Simulation into design proces of na architecture practice. Seventh International IBPSA Conference. Rio de Janeiro, Brazil. 2001. Disponível em: <http://www.ibpsa.org/proceedings/BS2001/BS01_0697_704.pdf>.

NAÇÕES UNIDAS BRASIL. Objetivos de Desenvolvimento Sustentável. Agenda 2030. Brasil, 2015. Disponível em:

< https://nacoesunidas.org/pos2015/ods11/>

OLGYAY, V., OLGYAY, A. Design with climate: bioclimatic approach to architectural regionalism. 1st Edition. Princeton University Press Princeton, Estados Unidos; 1963.

OSTERGARD, T.; JENSEN, R. L.; MAAGAARD, S. Building simulations supporting decision making in early design - A review. Renewable \& Sustainable Energy Reviews, 61 (August), 187-201. Aalborg University, Dinamarca. 2016.

PETERS, B., PETERS, T. Computing the Environment: Digital Design Tools for Simulation and Visualisation of Sustainable Architecture. 1st Edition. John Wiley \& Sons Ltd. Chinchester, UK. 2018.

RUUSKA, A., HÄKKINEN, T. Material efficiency of building construction. Buildings 4, 266-294; doi:10.3390/buildings4030266. Traduzido pelo autor, 2004.

TORCELLINI, P.; PLESS, S.; DERU, M. Zero Energy Buildings: A critical look at the Definition. Conference Paper NREL/CP-550-398333, Califórnia, US. 2006.

Disponível em: <https://www.nrel.gov/docs/fy06osti/39833.pdf>.

SUDBRACK, L. O. Casa Zero: Diretrizes de projeto para casas pré-fabricadas de balanço energético nulo em Brasília. 2017.242 f. Dissertação (Mestrado em Arquitetura) Faculdade de Arquitetura e Urbanismo, Universidade de Brasília, Brasília, 2017. 